\title{
Constraining star formation timescales with molecular gas and young star clusters
}

\author{
Kathryn Grasha ${ }^{1}$ and Daniela Calzetti ${ }^{2}$ \\ ${ }^{1}$ Research School of Astronomy and Astrophysics, Australian National University, Canberra, \\ ACT 2611, Australia \\ email: kathryn.grasha@anu.edu.au \\ ${ }^{2}$ Astronomy Department, University of Massachusetts, Amherst, MA 01003, USA \\ email: calzetti@astro.umass.edu
}

\begin{abstract}
Star formation provides insight into the physical processes that govern the transformation of gas into stars. A key missing piece in a predictive theory of star formation is the link between scales of individual stars and star clusters up to entire galaxies. LEGUS is now providing the information to test the overall organization and spatial evolution of star formation. We present our latest findings of using star clusters from LEGUS combined with ALMA CO observations to investigate the transition from molecular gas to star formation in local galaxies. This work paves the way for future JWST observations of the embedded phase of star formation, the last missing ingredient to connect young star clusters and their relation with gas reservoirs. Multi-wavelength studies of local galaxies and their stellar and gas components will help shed light on early phases of galaxy evolution and properties of the ISM at high-z.
\end{abstract}

Keywords. galaxies: star clusters, galaxies: stellar content, ISM: clouds, ISM: structure

\section{Introduction}

The process of star formation is a critical pillar in our understandings of the foundations of galaxy evolution. Despite this, the connection between the local processes at scales of individual molecular clouds, stars, and clusters with multi-kpc galactic scales remains ill-understood. Star clusters, identifiable in galaxies up to $\sim 100 \mathrm{Mpc}$, provide a direct observational signature of the star formation process.

Within this framework, the investigation of the spatial relation between young star clusters and their natal molecular gas provide an important tool to connect local scales of star formation with global scaling relations between star formation and gas reservoirs of entire galaxies (Kennicutt 1998). The path connecting the structure of galaxies and their ISM, the properties of their molecular clouds, and the characteristics of the resulting stellar clusters are virtually uncharted. The importance of our physical understanding of resolved star formation extends beyond local galaxies and will help shed light on the evolution of star formation and its subsequent impact on galactic evolution from clumpy galaxies at high redshift to the smoother disks seen today.

\section{Overview}

Using young star clusters from the Legacy ExtraGalactic UV Survey (LEGUS; Calzetti et al. 2015), an HST Treasury program of 50 nearby galaxies, and CO molecular gas, we are able to constrain the timescale for the association of star clusters and their natal molecular clouds in two nearby spiral galaxies, NGC 5194 (Figure 1; Grasha et al. 2019; Schinnerer et al. 2013) and NGC 7793 (Grasha et al. 2018). 

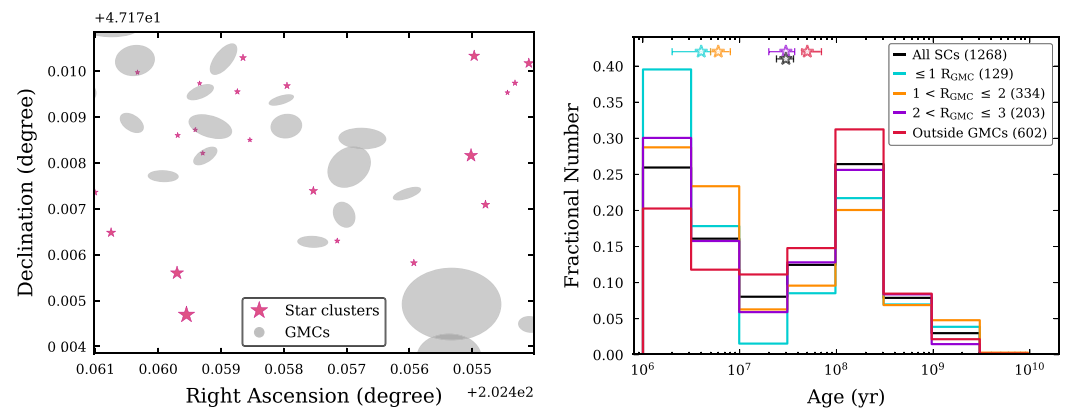

Figure 1. Left: Star clusters (pink stars) and GMCs (gray ellipses) in NGC 5194. There is a statistical tend for younger star clusters (smaller stars) to be located within the vicinity of molecular gas and older star clusters (larger stars) to be isolated. Right: Age distribution of the star clusters in bins of distance to their nearest GMC. Star clusters still located within a GMC show ages of $4 \mathrm{Myr}$ (teal) whereas isolated star clusters show ages > $50 \mathrm{Myr}$ (red).

We measure the distance between each star cluster to a GMC to constrain the timescale and velocity for star clusters to separate from their clouds. We find the expected trend for younger star clusters to still reside in areas where GMCs still live (Figure 1), with star clusters losing their association with their GMCs after $~ 2-3$ Myr in NGC 7793 and 4-6 Myr in NGC 5194. These timescales are a combination of stellar movement and cloud erosion via feedback and suggest that there may be environmental impacts on how long star clusters remain embedded in their natal clouds.

\section{Implications}

Multi-tracers of gas. The key to understanding the conversion of gas into stars likely cannot be found in a single gas phase: the transition from atomic, to molecular, to dense gas, and to stars, and the underlying mechanisms that maintain the balance between the different gas phases are ultimately what drives and controls the star formation cycle.

A pivotal, ill-studied component in this transition is the dense gas phase of the ISM, highlighting the importance for incorporating the critical role of dense gas in spatially linking gas to regions of newborn stars with facilities such as the Large Millimeter Telescope and ALMA. The different timescales for CO-cluster association versus that of dense gas are crucial for a quantitative description of the evolution of young stellar populations and the co-evolution of star formation, stellar populations, and gas.

Mechanisms that drive the star cluster-molecular gas timescale. Star formation is understood as a consequence of dense gas concentrated within giant molecular clouds (GMCs) that undergo fragmentation and other feedback processes that act to suppress star formation (Krumholz 2014). The short timescales we derive indicate that feedback and stellar wind dominate over other effects such as supernovae (Dale et al. 2014). The exact timescale for the expulsion of gas has broad ranging implications from constraining multiple stellar generations in globular clusters (Krause et al. 2013) to the reionization of the Universe (Ma et al. 2015).

\section{References}

Calzetti, D. et al. 2015, AJ, 149, 51

Dale, J. et al. 2014, MNRAS, 442, 694

Grasha, K. et al. 2018, MNRAS, 481, 1016

Grasha, K. et al. 2019, MNRAS, 483, 4707

Kennicutt, R. C. 1998, ApJ, 498, 541 
Krause, M. et al. 2013, A\&\&A, 550, A49

Krumholz, M. 2014, Phys. Rep., 539, 49

Ma, X. et al. 2015, MNRAS, 453, 960

Schinnerer, E. et al. 2013, ApJ, 779, 42 